\title{
Analysis of Multimedia Teaching Path of Popular Music Based on Multiple Intelligence Teaching Mode
}

\author{
Dongfang Wang (iD \\ The Department of Music, XinXiang University, XinXiang 453003, China \\ Correspondence should be addressed to Dongfang Wang; wangdongfang@xxu.edu.cn
}

Received 18 November 2021; Revised 15 December 2021; Accepted 24 December 2021; Published 28 January 2022

Academic Editor: Qiangyi Li

Copyright (c) 2022 Dongfang Wang. This is an open access article distributed under the Creative Commons Attribution License, which permits unrestricted use, distribution, and reproduction in any medium, provided the original work is properly cited.

Pop music multimedia is one of the popular digital pop music types. Based on the multiple intelligences teaching model, a multimedia multiple intelligences teaching method of popular music is proposed. This method not only analyzes the characteristics of pop music in detail, but also fully considers other important characteristics of pop music. It teaches college students multimedia, purifies their hearts, improves their personality, cultivates their innovative consciousness, and promotes their healthy growth. In this paper, the multiple intelligences teaching model is introduced into the process of pop music multimedia teaching path. In the stage of music audio segmentation, the deep belief network algorithm is used to accurately carry out music multimedia teaching. Finally, the experimental analysis results show that the integration of pop factors into music teaching, the combination of pop music and quality education, and the creation of music that is suitable for students' personality characteristics and the needs of aesthetic development can better serve the needs of students' quality improvement.

\section{Introduction}

With the continuous development of Internet technology and the improvement of technological progress, different Internet-based popular music multimedia began to emerge [1]. As one of the more popular types of digital pop music, the teaching and recognition of multiple intelligences of popular music multimedia have become the focus of domestic scholars' research. However, how to obtain the specific content source from the initial popular music multimedia data lacking the definition of popular music content has become a huge challenge for the current popular music multimedia multi-intelligence teaching, because the popular music multimedia signal belongs to a way of chronological order, the multiple intelligences teaching mode can be used according to its invisibility. At present, other classification methods are relatively simple, and it is not accurate to obtain the characteristics of popular music multimedia.

The multiple intelligence teaching model is applied to the automatic classification process of popular music multimedia in this paper. This method can use the lyrics, word frequency, content, and meaning of popular music multimedia as current prior knowledge in the automatic classification process of popular music multimedia according to the features of automatic lyrics of popular music multimedia. The information gain method is used to obtain popular music characteristics of popular music multimedia content; this mode is used to limit the weight of lyrics and the semantic information of popular music, integrate lyrics with high meaning similarity, and construct various types of popular music multimedia teaching models.

\section{Multiple Intelligence Teaching Mode}

When multiple intelligences teaching usually uses a given sequence of observations $O=o_{1}, o_{2}, \ldots, o_{T}$, a certain $\lambda=(\mathrm{A}$, $\mathrm{B}, \pi)$ parameter is used to multimedia, ensuring that the value of $P(O \mid \lambda)$ can reach the maximum value in this method.

(1) According to the related definitions of forward probability and backward probability, we know 
$P(O \mid \lambda)=\sum_{i=1}^{N} \sum_{j=1}^{N} \alpha_{t}(i) a_{i j} b_{j}\left(o_{t+1}\right) \beta_{t+1}(j), \quad 1 \leq t \leq T-1$.

When $P(O \mid \lambda)$ being used reaches the maximum value, the training sequence of each experiment is limited, so the best method of estimating parameters cannot be achieved. In this case, the Baum-Welch algorithm uses recursion thought with $P(O \mid \lambda)$, the part is very large, and finally the model parameter $\lambda=(A, B, \pi)$ is obtained.

(2) The revaluation formula of the Baum-Welch algorithm is derived by recursion as

$$
\begin{aligned}
\bar{\pi}_{i} & =\xi_{1}(i), \\
\bar{a}_{i j} & =\frac{\sum_{t=1}^{T-1} \xi_{t}(i, j)}{\sum_{t=1}^{T-1} \xi_{t}(i)}, \\
\bar{b}_{j k} & =\frac{\sum_{t=1 \text { and } O_{t}=V_{k}}^{T} \xi_{t}(j)}{\sum_{t=1}^{T} \xi_{t}(j)} .
\end{aligned}
$$

Among them, $\xi_{t}(i, j)$ represents the given training sequence $\mathrm{O}$ and model parameters. $\Lambda$, the state of multiple $\mathrm{v}$-chains at time $t$ and the time $t+1$ is the state probability, $\theta_{i} \theta_{i} \sum_{t=1}^{T-1} \xi_{t}(i)$ represents the expected value of the number of transitions from state $\theta_{t}$ to state $\theta_{i}$.

Define the auxiliary function as

$Q(\lambda, \bar{\lambda})=\frac{1}{P(O \mid \lambda)} \sum_{\text {all } S} P(O, S \mid \lambda) \ln P(O, S \mid \bar{\lambda})$.

(3) Among them, $\lambda$ is the original model parameter, $\bar{\lambda}$ represents the model parameter to be solved $\bar{\lambda}=(\bar{A}, \bar{B}, \bar{\pi}), O$ represents the observation sequence used for training, $O=o_{1}, o_{2}, \ldots, o_{T}$, and $S$ is a certain state sequence $S=q_{1}, q_{2}, \ldots, q_{T}$.

The multi-intelligence education model can not only be used to find a good enough state transition path, but also quickly calculate the output probability corresponding to the path. Meanwhile, the calculated amount required by the method of using the Markov model to calculate the output probability is much less than that in the total probability formula.

$\delta_{t}(i)$ is defined as the maximum probability $o_{1}, o_{2}, \ldots, o_{t}$ produced by following a path $q_{1}, q_{2}, \ldots, q_{t}$ at time $t$ and $q_{1}=$ $\theta_{i} \quad$ which is $\delta_{t}(i)=\max _{q_{1}, q_{2}, \ldots, q_{t-1}} P\left(q_{1}, q_{2}, \ldots, q_{t}, \quad q_{1}=\theta_{i}\right.$,
$\left.o_{1}, o_{2}, \ldots, o_{t} \mid \lambda\right)$.

The recursive form of the multiple intelligences teaching is as follows.

(1) Initialization:

$$
\begin{aligned}
\delta_{t}(i) & =\pi_{i} b_{i}\left(o_{1}\right), \quad 1 \leq i \leq N, \\
\psi_{1}(i) & =0 .
\end{aligned}
$$

(2) Recursion:

$$
\begin{aligned}
& \delta_{t}(j)=\max _{1 \leq i \leq N}\left[\delta_{t-1}(i) a_{i j}\right] b_{j}\left(o_{t}\right), \quad 2 \leq t \leq T, 1 \leq j \leq N, \\
& \psi_{t}(j)=\underset{1 \leq i \leq N}{\arg \max }\left[\delta_{t-1}(i) a_{i j}\right], \quad 2 \leq t \leq T, 1 \leq j \leq N .
\end{aligned}
$$

(3) End:

$$
\begin{aligned}
P^{*} & =\max _{1 \leq i \leq N} \delta_{T}(i), \\
q_{T}^{*} & =\underset{1 \leq i \leq N}{\arg \max } \delta_{T}(i) .
\end{aligned}
$$

(4) Find the state sequence:

$$
q_{t}^{*}=\psi_{t+1}\left(q_{t+1}^{*}\right), \quad 1 \leq t \leq T-1 .
$$

Among them, $\delta_{t}(i)$ represents the probability of accumulating the output value of the $i$-th state at time $t, \psi_{t}(i)$ represents the continuous state parameter of the $i$-th state at time $t, q_{t}^{*}$ is the state at time $t$ in the optimal state sequence, and $P^{*}$ is the final output probability.

The multiple intelligence teaching model is a multilayered multiple intelligence teaching model that can automatically learn from above and below. This is the accumulation of multiple restricted Boltzmann machines (RBM). Its structure is shown in Figure 1, and every two adjacent layers constitute a restricted Boltzmann machine. From bottom to top, the hidden layer of the previous RBM is the visible layer of the next RBM. The output of the previous $\mathrm{RBM}$ is the input of the next RBM, so as to search to the last layer for each level.

Through multimedia teaching and learning, the link weights and node offsets of each layer are obtained, and the network initialization is completed. The inverse conduction algorithm (BP) is used to fine-tune the deep trust network model monitored from top to bottom. Overcome the shortcomings of local optimization and long search time. Although the performance of the deep belief network model shows strong characteristic learning capabilities, from the above principles, Internet search requires a large amount of sample data to generate more parameter values $[2,3]$. On the other hand, based on the popular music recommendation search problem, which lies in lack of a large amount of sample data, it is found that the generation of a large number of parameters takes a long time, which is not good for practical applications.

Different from traditional machine learning methods, the characteristic of the multimedia education model is the original time-domain characteristics of the input signal, which automatically learns the input signal through hierarchical multimedia education. The general time domain features are the short-term average amplitude difference, short-term energy, short-term autocorrelation coefficient, and so on. In this article, short-term energy is used as the original input characteristics of the deep multiple intelligence teaching model. The short-term energy can reflect the process of the energy of the sound signal changing over time. 


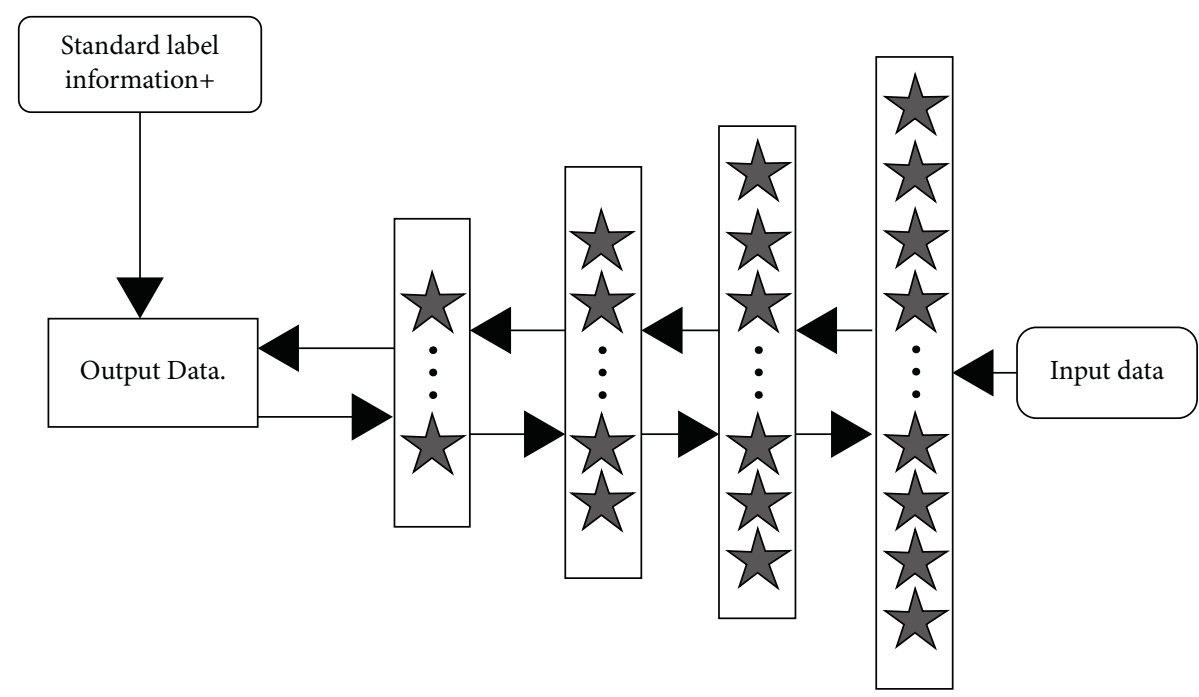

Figure 1: Multiple intelligence teaching model.

Unvoiced and voiced syllables can be clearly distinguished by short-term energy characteristics. Set the popular music signal as $x(n)$; the average energy of that short time is as follows:

$$
\begin{aligned}
E_{n} & =\sum_{m=-\infty}^{\infty}[x(n) w(n-m)]^{2} \\
& =\sum_{m=-\infty}^{\infty} x(m)^{2} h(n-m) \\
& =x(m)^{2} * h(n) .
\end{aligned}
$$

However, the characteristic dimension of the original time domain is high, in which there is a lot of redundancy and noise. Therefore, the input search data requires lowdimensional processing. The method of Principal Component Analysis (PCA) in this paper investigates the correlation between multiple variables by means of multiple statistical methods. It is studied that the internal structure of multiple variables is clarified through several main components through several principal components. After the multimedia teaching data is processed by dimensionality reduction, it is input into the multiple intelligence teaching mode for multimedia teaching, automatically learning the characteristic information of the data, and finally processing the output data with the Softmax multimedia education path function commonly used in deep learning. Complete the task of multimedia teaching path. Specifically, the multimedia teaching path structure of popular music based on multiple intelligence teaching mode is shown in Figure 2.

A multiple intelligence teaching model corresponding to the shortcomings of the original network model is proposed in this paper, which is different from the original multiple intelligence teaching model. As shown in Figure 3, the stacked and downsampling layers in the network with deep beliefs join the multiple intelligence teaching model together. The second layer of the multiple intelligence education model has 5 nodes. There are 11 parameters that require multimedia teaching when there are 3 nodes on the third layer using all connections. With quota sharing and sampling, there are 4 parameters that require multimedia teaching. The output of the convolutional layer acts as a hidden layer node of the next Boltzmann machine with restrictions.

\section{Problems Existing in the Development of Multimedia Teaching of Popular Music in the New Era}

3.1. Popular Music Needs to Be Strengthened in the Combination of Nationality and Sense of the Times. Nationality and the sense of the times are the wings and two wheels of popular songs, supporting each other. The lack of national flavor leads to the lack of life elements in popular music; due to the lack of modernity, the influence and dissemination of popular music will be greatly affected, especially because the characteristics of popular music in China are different $[4,5]$. Currently, according to statistics, there are more than 300,000 pieces of popular music. According to national characteristics, various types of popular music are continuously produced. Popular music also has national characteristics. The tune and style of songs can express national characteristics. This shows that the different regional characteristics of popular music also enrich popular music, because different ethnic groups have different music styles, but they also have the characteristics and aesthetic characteristics of fashionable music from the perspective of dialectics; it is undeniable that the existing popular music is also influenced by the foreign culture, such as rock, jazz and other music. Because of the unique style and compact rhythm of popular music, it is deeply loved by young people. In recent years, young people have lost their interest in national music in an unknown way. Only on a few occasions, because popular music pursues capital profits, they occupy the leading position of mainstream music and gather in popular songs. On the contrary, for national music with low economic value, through appropriate injection of popular elements of the times, it will be praised by the public. 


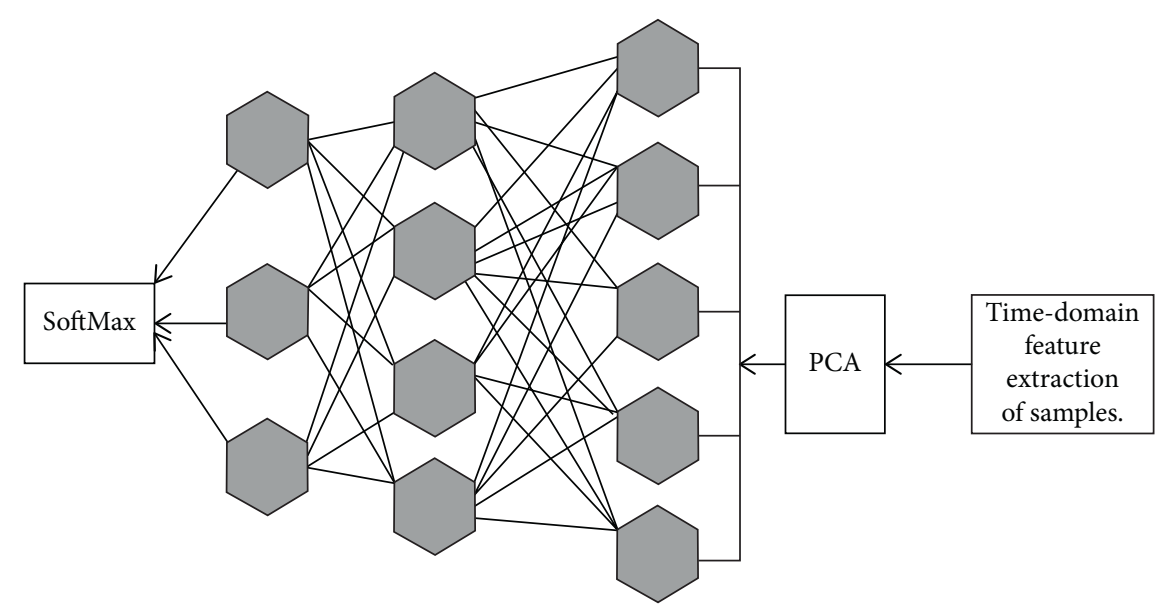

FIgURE 2: Pop music multimedia teaching path based on multiple intelligences teaching modes.

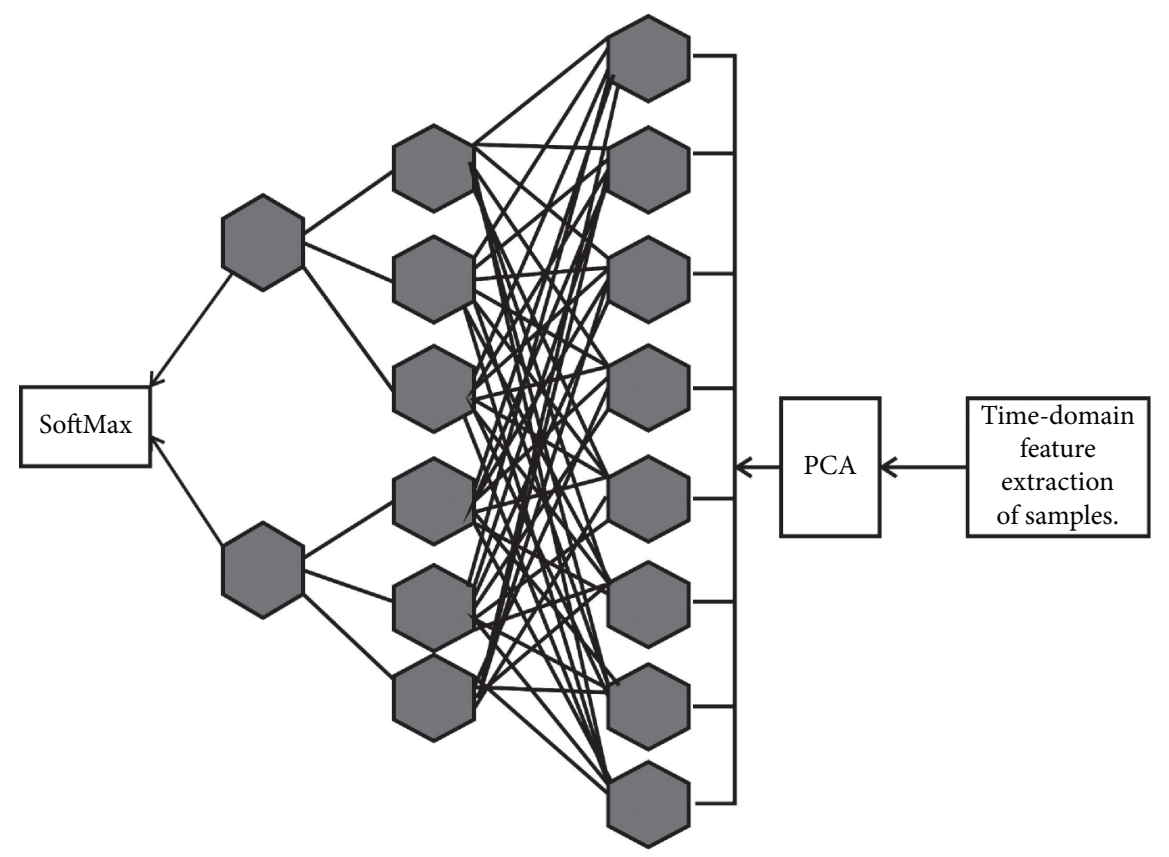

FigURE 3: Extraction of samples based on multiple intelligences teaching.

3.2. Popular Music Development and Multimedia Teaching Need to Be Strengthened. Ethnic groups are generally located in remote areas. Due to the underdeveloped economy, the investment of traditional music and multimedia in education is relatively insufficient. Taking Guangxi Zhuang Autonomous Region as an example, Zhuang people's residences are concentrated in Laike, Hechi, Baise, Chongzuo, and other places, forming a relatively closed living environment and a realistic state of human existence. At the same time, it also forms the originality and uniqueness and irreplaceability of the Zhuang national culture [6]. Guangxi is known as Song Sea. Song Sea: there is a traditional folk song festival every year, but the singing activities are generally only held in the village. The infrastructure is weak, and the activities are held in small scale. Lack of innovative forms: because of the weak funding guarantee, there is also a big gap in the education of popular music and multimedia. It is understood that there is little basic multimedia education in the local area, and traditional popular music courses have been opened. Popular music equipment and popular music teachers are seriously inadequate. Without basic music facilities, even very excellent musicians cannot play beautiful melody. Insufficient investment has severely restricted the development of popular music and multimedia teaching.

3.3. The Shortage of Multimedia Teaching Talents for Popular Music Needs to Be Strengthened. At present, the structure of the teaching staff of popular music multimedia education is unreasonable. First, the total amount is insufficient, and there are few talents specializing in multimedia education of 
popular music. Second, the quality is not high. Many music teachers are familiar with popular music. They have little understanding of popular music. Third, due to the poor structure, popular music multimedia education was carried out in ethnic areas. It is developed appropriately in the city, but poorly in the countryside, appropriately in the field of higher multimedia education, and poorly in the field of basic multimedia education. Fourth, the reserves are insufficient. These high schools have trained pop music talents. Because of employment and entrepreneurship and other reasons, the Matthew effect of insufficient continuity, insufficient charm, and insufficient talent accumulation has been brought about. In addition, popular music multimedia education is different from general music multimedia education. Another important feature is that popular music multimedia education relies on national folk music artists $[7,8]$. National folk artists are deeply influenced by local customs and cultural traditions. They have deep artistic attainments and shelter in the folk. Underground treasure house is also an important disseminator of popular music. Now, due to the lack of national attention to folk artists, the shadow of folk artists cannot be seen in many major performance venues. It is hard to see folk artists from ethnic groups enter the campus and the classroom and give practical teaching. Folk artists of a nation are old and have deep knowledge of music because they have not received corresponding attention. Many popular music types disappeared with the death of these folk artists. This is not only a regret for music inheritance, but also a loss of national culture.

\section{New Features under the Multiple Intelligence Teaching Mode}

4.1. Popularity. Popularity is a relatively distinctive feature in modern mass aesthetic culture. In order to meet the aesthetic needs of the masses in the development process, the multi-intelligence education model continuously innovates expression forms and singing methods, pays more attention to the lives of the masses, and expresses the feelings of the masses with musical works. Strike a chord. In addition, popular music works have also made many people wiser. The popularization of the multi-intelligence education model is mainly manifested in the following two aspects. One is the popularization of the content of the multi-intelligence education model [9]. The traditional and diverse intelligent education models are mostly works that sing the theme of the motherland and the form of opera. With the development of the times and the changes in people's concepts and aesthetics, the singing in the multiple intelligence teaching mode can be an ensemble, a counterpart, a combination with dance, a combination of bel canto, and a collocation with popular music. You can also add high-tech lighting, sound, and other Western musical instruments, such as guitars and basses. The songs of multiple intelligent education modes are very rich. For example, Chinese bass drums are incorporated into the "New Ambuscade from all Sides." In addition to guitars and basses and other musical instruments, electroacoustic technology is used to perform the tension and courage of the drama.
4.2. Entertaining. The multiple intelligence teaching mode makes the artistic expression of the multiple intelligence teaching model full of entertainment to a certain extent. Films, TV works, video products, etc. are used to fully demonstrate the commercial and entertaining nature of the multi-intelligence education model. The entertaining nature of the multi-intelligence education model also reflects the public's listening needs and consumer psychology. Especially for modern young people who are under great pressure in life, study, and work, the entertainment of multiple intelligent education modes fully meets the spiritual needs of pursuing public stimulation and venting pressure $[10,11]$. The multiple intelligence teaching model has some insurmountable shortcomings in data multimedia teaching. In order to solve this problem, it is necessary to try to reduce the number of parameters of multimedia education in the multifunctional education mode. The general technology is the sharing method. Sharing right is to adopt the idea of layers that are folded in the deep belief network. Different from the complete connection and parameter sharing of the general multiple intelligence teaching model, the deep belief network has the characteristics of partial connection and rights sharing. Weight sharing means that the connections of multiple nodes in the hierarchy share the same set of parameters. The feasibility study of sharing parameters is mainly related to many objects in life. For example, the signal of each frame of pop music is not isolated but continuously plays the common role of each frame. Deliver popular music to the audience. Therefore, a deep belief network can be combined with a multiple intelligence teaching model. If quota sharing technology is used, the number of parameters will be significantly reduced, which can reflect popular music to convey the essence of popular music. It is of great significance.

4.3. Feasibility. Popular music education not only is related to students' lives and all aspects of life, but also can bring them aesthetic enjoyment. And it will stimulate their pursuit of truth, goodness, and beauty, increase knowledge, broaden their horizons, and promote their physical and mental health. The integration of multimedia teaching in music education makes teaching more appealing and attractive, richer in images and emotions, and easier for students to accept, so that their comprehensive quality can be improved unconsciously. The fundamental purpose of developing music multimedia education is to cultivate college students' aesthetic ability and music appreciation ability, establish correct and positive values and outlook on life, comprehend the poetic imagery beauty of music and popular music, and be nurtured. Create a sound personality. Music education is an important carrier of quality education, a basic form of multimedia education, and an important form of education. The effective development of multimedia education in the process of music education plays a very important role in promoting the effective implementation of quality education and aesthetic education. At present, with the advancement of science and technology and the development of Internet technology, many multimedia entertainment platforms all 
provide background online digital popular music recommendation and multimedia teaching services according to users' preferences, in order to facilitate the multimedia teaching of popular music by users. With the continuous expansion of popular music database, the traditional music multimedia teaching mode can no longer meet the actual needs of users according to the data information of popular music names, songwriters, etc. [12]. Therefore, it is urgent to use popular music multimedia teaching path technology to provide users with simple and smart pop music selection mode. It is a way of conveying artistic feelings. Popular music is also one of the modes that can best embody the characteristics of music. According to the statistics of an existing popular music website, popular music is a brand that users often perform calibration. The popular music multimedia teaching path can be considered from the perspective of popular music perception and can provide users with convenient services.

4.4. Multimedia Teaching Path of Popular Music under the Multiple Intelligence Teaching Mode. Popular music multimedia education has achieved a series of research results. The relationship between the query phrase and the document is modeled through the Bellius network, and the time and text characteristics of the document are considered. A structured representation method of popular music information is proposed in this paper. This method can bundle popular music through the text structure. Each category has specific information characteristics. These features can improve various intelligent education effects in popular music multimedia. In addition to the research on the multiple intelligence education of popular music multimedia texts, there is also the research on the multiple intelligence education methods of popular music multimedia user information analysis. In addition, the multimedia teaching of popular music also includes the multiple intelligences teaching of the subject words of popular music. The multiple intelligences teaching for the subject words will be used to carry out multiple intelligences' education for languages with such signs. Users usually import subject words that represent popular music topics. Therefore, the multiple intelligence education of subject words can directly obtain popular music related to the content [13]. Noise and redundant information are filtered in social media to obtain high-quality information. A high-quality pop music multimedia extraction framework is proposed based on kernel principal analysis and wavelet transform in this paper, and a high-quality information extraction algorithm is designed based on multifeature fusion. Figure 4 shows the construction diagram of "three hundred" actual ability training.

The multi-intelligence education model is a machine learning algorithm that effectively integrates relevant characteristics into an ordered model [14]. In the education of diversity and multiple intelligences, in addition to the relevance of popular music and search terms, the similarities in popular music must also be considered. A variety of intelligent education modes are adopted to solve the problem of diverse and multiple intelligent education in popular music multimedia. Through the mechanical learning method, it has produced a lot of influence on the relevance of popular music and search terms and the similarity of popular music and transformed it into the features for training and test. The features are combined in different ways, and the effectiveness of the features is verified through the ranking effect. Figure 5 shows the framework of diversity ranking learning in popular music multimedia.

First, given a set of query words $Q=\left\{q_{1}, q_{2}, \ldots, q_{n}\right\}$, each query word $q$ has a corresponding pop music set $T_{i}=\left\{\right.$ tweet $_{i 1}$, $_{\text {tweet }}{ }_{i 2}, \ldots$, tweet $\left._{i m}\right\}$. Each piece of popular

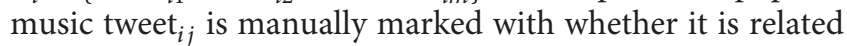
to the corresponding query term $q_{i}$ and its corresponding subtopic (query intent), and the standard answer $\operatorname{rank}_{i}$ for sorting the popular music collection $T_{i}$ is generated through this marked information. Each query word $q$ has a tweet ${ }_{i j}$ corresponding to pop music collection $T_{i}=\left\{\right.$ tweet $_{i 1}$, tweet $_{i 2}, \ldots$, tweet $\left._{i m}\right\}$. A series of features used to measure the correlation between each piece of popular music in the popular music collection $T_{i}$ and the query word $q_{i}$ and measure the similarity between each piece of popular music in the popular music collection $T$ are designed and extracted to form a feature vector, vector $_{i j}$. Assuming that the ranking function in the diversity ranking is $f$, the goal of diversity ranking learning is to obtain the optimal ranking function $f$ through manually labeled training data.

For the new query $q$ and its corresponding pop music set $T$, the same features are extracted to form a feature vector adopted, and the sorting function $f$ is used to generate the final diversity and multiple intelligent education results. We represent the definition formula (1) of the ranking function in the diversity ranking learning.

$$
f\left(x_{j}^{(i)}, r_{j}^{(i)}\right)=w_{r}^{T} x_{j}^{(i)}+w_{d}^{T} h\left(r_{j}^{(i)}\right) .
$$

Among them, vector $_{i j}$ represents the correlation feature vector between the query $q_{i}$ and the corresponding popular music tweet ${ }_{i j}, r_{j}^{(i)}$ represents the similarity feature matrix between popular music tweet ${ }_{i j}$ and the popular music set ranked in front of it, and the function $h$ transforms the similarity feature matrix into feature vector by averaging multiple values corresponding to each feature. $w_{r}^{T}$ and $w_{d}^{T}$, respectively, represent the weights of the correlation feature vector and the similarity feature vector.

The multiple intelligence teaching model is a new tool to solve the quality evaluation of popular music multimedia teaching by means of optimization methods and is especially suitable for the study of evaluation problems. Its essence is based on the multiple intelligence teaching model in popular music multimedia teaching path analysis, to establish a popular music multimedia teaching path analysis method using linear function hypothesis space.

Assuming the analysis of popular music multimedia teaching path, the popular music multimedia set is $\left(x_{i}, y_{i}\right), i=1,2, \ldots, n x \in R^{d} y \in\{-1,+1\}$. In the $d$ dimensional space, the general form of popular music multimedia teaching path analysis function is $g(x)=w \cdot x+b$, and the specific classification surface equation is

$$
w \cdot x+b=0 \text {. }
$$




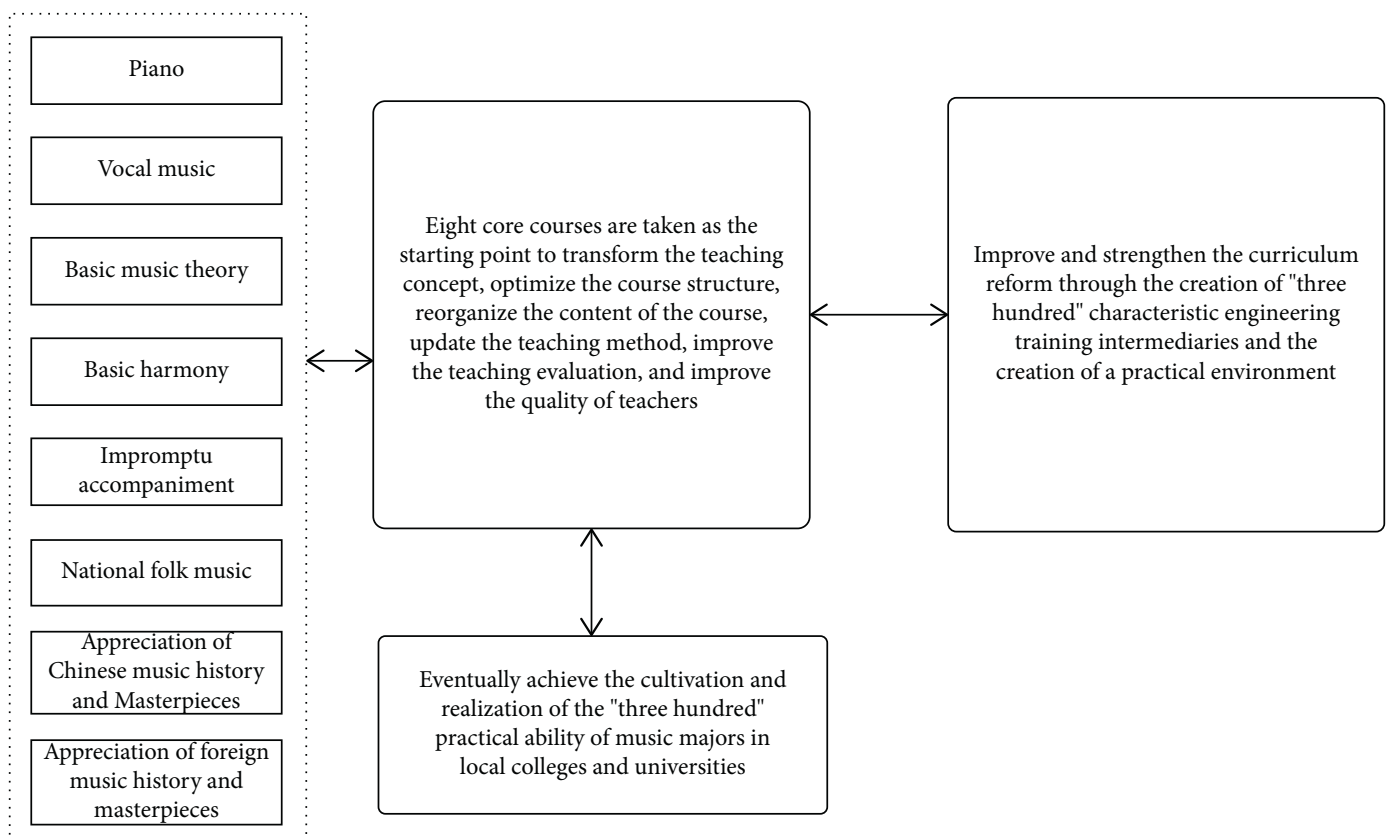

FIgURE 4: “Three hundred” practical ability construction diagram.

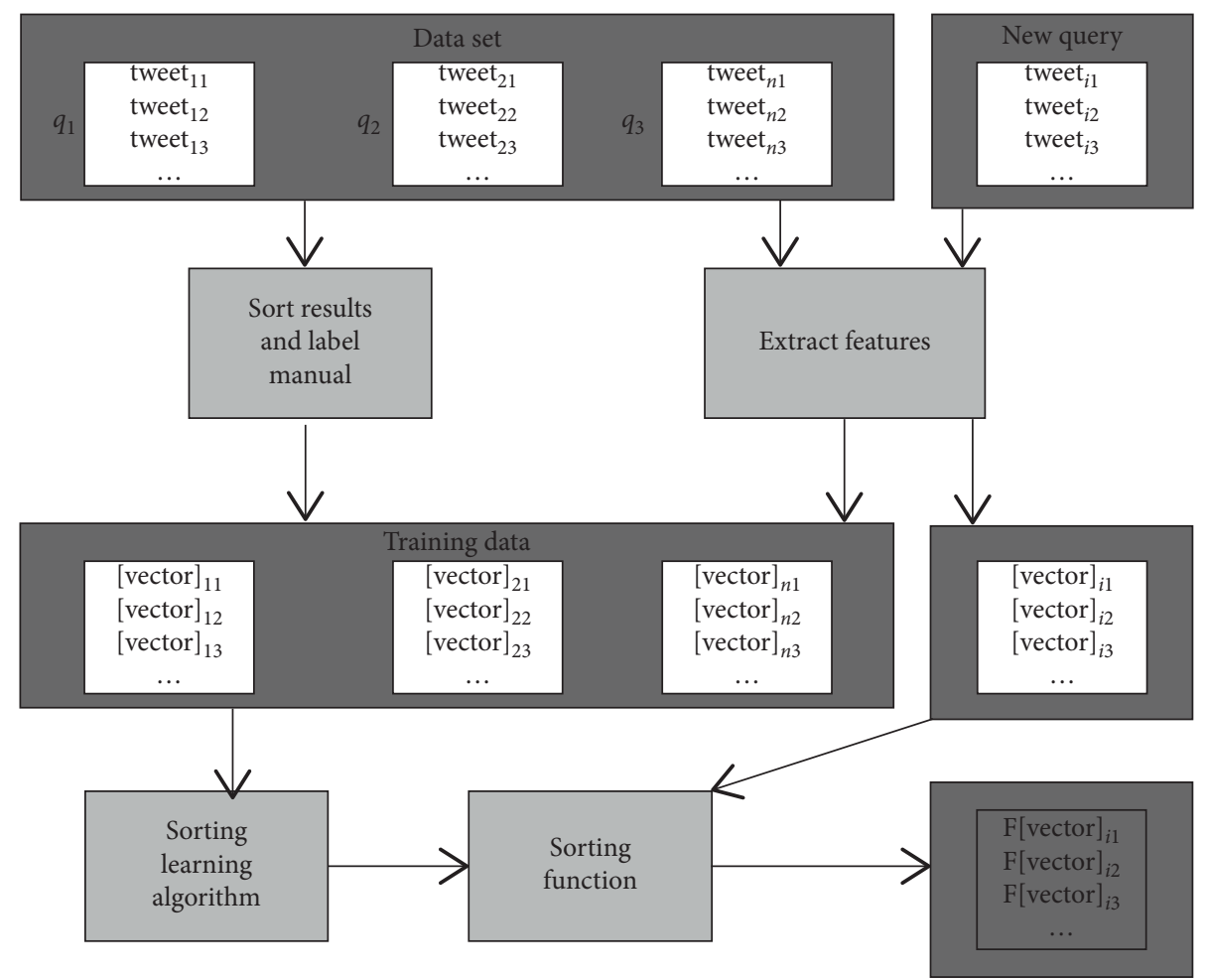

FIgURE 5: Diversity ranking learning framework.

To ensure that the classification surface of all popular music multimedia can be classified correctly, it must be satisfied:

$$
y_{i}[(w \cdot x)+b-1] \geq 0, \quad i=1,2, \ldots, n .
$$

As mentioned above, if the above conditions are met, the smallest classification surface is the best classification surface. The matching of string similarity can convert the solution problem of optimal classification into the following constraint optimization problem; that is, the 
calculation function $\|w\|^{2}$ is satisfied under the restraint in formula (11).

$$
\begin{aligned}
\varphi(w) & =\frac{1}{2}\|w\|^{2} \\
& =\frac{1}{2}(w \cdot w) .
\end{aligned}
$$

The minimum value: this problem is further transformed into Lagrange function:

$$
\left.L(w, b, a)=\frac{1}{2}(w \cdot w)-\sum_{i=1}^{n} a_{i}\left\{y_{i}\left[w \cdot x_{i}\right)+b\right]-1\right\} .
$$

The optimal classification function obtained by solving is

$$
\begin{aligned}
f(x) & =\operatorname{sgn}\{(w * \cdot x+b *\} \\
& =\operatorname{sgn}\left\{\sum_{i=1}^{n} a_{i}^{*} y_{i} \cdot\left(x_{i} \cdot x\right)+b *\right\},
\end{aligned}
$$

where sgn () is a symbolic function. In the case of strict analysis of popular music multimedia teaching path in popular music multimedia, it can be processed according to formulas (10) (14); however, when popular music multimedia cannot conduct the analysis of popular music multimedia teaching path, slack variables need to be introduced; namely, the condition is satisfied by adding a slack variable $\xi_{i} \geq 0$ in (11), so that (10) can be used.

$$
y_{i}[(w \cdot x)+b-1]+\xi_{i} \geq 0, \quad i=1,2, \ldots, n .
$$

The corresponding objective function is also transformed into the minimum value:

$$
\phi(w, \xi)=\frac{1}{2}\|w\|^{2}-C\left|\sum_{i=1}^{n} \xi_{i}\right| .
$$

As a typical social media platform, popular music multimedia has its rich user information, which may help solve the problem of popular music multimedia education. Therefore, it is necessary to consider the characteristics of users among popular music publishers. The user characteristics of popular music performers include the geographic location of the user, whether the user is authenticated, the user language, the number of popular songs released by the user, the number of friends of the user, the number of followers of the user, and the number of times the user is grouped into other users.

4.5. Experiment and Result Analysis. 26 songs and 5 kinds of popular music are selected in different styles on popular music websites, and a total of 200 pieces of popular music are provided for this paper. Perform preprocessing, feature extraction, multiple intelligence calculations, and angle cosine cluster analysis for the abovementioned popular music.

In the preprocessing stage, segmentation and subframe processing are performed first. For popular music, its local segment is similar to the entire popular music, and a popular music segment needs to be truncated with a segment length

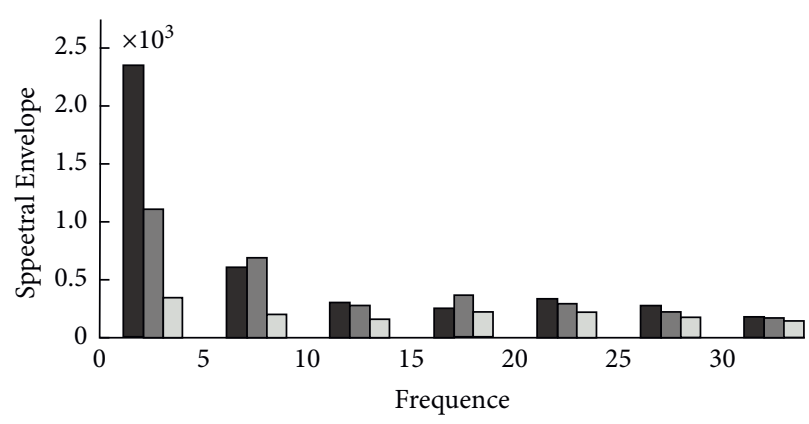

Classical Music 1

Pop Music 2

$\square$ Classical Music 3

Figure 6: Spectrum envelope of 3 pieces of popular music.

$$
\begin{aligned}
& \quad d_{\text {sum; }} ; \\
& \text { for }(i=1: n)\{ \\
& d_{i}=a b s\left(x_{i}-y_{i}\right) \\
& \min =i ; \text { for }(=2: n)\{ \\
& d_{j}=a b s\left(x_{i}-y_{j}\right) ; \\
& \text { if } d_{j}<d_{i} ; \\
& d_{i}=d_{j} ; \\
& \min =j ; \\
& \} \\
& \} \\
& d_{\text {sum }}=d_{\text {sum }}+d_{i} ;
\end{aligned}
$$

Algorithm 1: Baum-Welch algorithm..

of about 3-5 minutes to reduce information redundancy and speed up the calculation. In this experiment, the initial popular music is divided into 15 segments, and $70 \%$ of the data of each segment is extracted as the signal processing data source.

In this paper, we choose to extract the time domain energy, frequency domain energy, spectral envelope, and MFCC features of the audio signal. If the triangle filter is selected, the center frequency is taken as M24. The spectral envelope is using formulas.

$$
f(m), \quad m=1,2, \ldots, M .
$$

The length produced by Hilbert transform is 4098. As shown in Figure 6, there are three different styles of popular music spectrum. The envelope shows a big difference in the spectral envelope diagram, especially in the low frequency part.

In this experiment, the abovementioned feature vector and feature vector are integrated to perform Hausdorff feature dimension calculation. Through frame segmentation and window processing, the simplified data segment still has many features. Therefore, for each feature of popular music, the first $20 \%$ is a 2056 -dimensional vector formed into a data set.

Before using sandwich cosine for clustering, it is necessary to regularize the obtained vector. For two popular 
TABLE 1: Multi-intelligence clustering result.

\begin{tabular}{|c|c|c|c|c|c|c|}
\hline \multirow{2}{*}{ Clustering cluster } & \multirow{2}{*}{ Total popular music } & \multirow{2}{*}{ Clustering result } & \multicolumn{4}{|c|}{ Style of popular music, } \\
\hline & & & M1 (sentimental) & M2 (passionate) & M3 (quiet) & M4 (lonely) \\
\hline \multirow{2}{*}{ Type 1} & \multirow{2}{*}{30} & $\mathrm{~T}$ & 21 & 24 & 20 & 18 \\
\hline & & $\mathrm{F}$ & $1 \mathrm{M} 34 \mathrm{M} 4$ & 0 & $2 \mathrm{M} 12 \mathrm{M} 3$ & $3 \mathrm{M} 13 \mathrm{M} 3$ \\
\hline \multirow{2}{*}{ Type 2} & \multirow{2}{*}{30} & $\mathrm{~T}$ & 21 & 23 & 19 & 24 \\
\hline & & $\mathrm{F}$ & $3 \mathrm{M} 4$ & $1 \mathrm{M} 12 \mathrm{M} 3$ & $5 \mathrm{M} 12 \mathrm{M} 4$ & $2 \mathrm{M} 3$ \\
\hline \multirow{2}{*}{ Type 3} & \multirow{2}{*}{30} & $\mathrm{~T}$ & 21 & 24 & 22 & 23 \\
\hline & & $\mathrm{F}$ & $2 \mathrm{M} 23 \mathrm{M} 3$ & $2 \mathrm{M} 4$ & $1 \mathrm{M} 12 \mathrm{M} 31 \mathrm{M} 42 \mathrm{M} 1$ 1M3 & \\
\hline \multirow{2}{*}{ Type 4} & \multirow{2}{*}{30} & $\mathrm{~T}$ & 16 & 24 & 23 & 21 \\
\hline & & $\mathrm{F}$ & $2 \mathrm{M} 23 \mathrm{M} 35 \mathrm{M} 4$ & $2 \mathrm{M} 4$ & $2 \mathrm{M} 11 \mathrm{M} 3$ & 2M1 3M4 \\
\hline
\end{tabular}

TABle 2: Statistics of the classification accuracy of the four popular music styles.

\begin{tabular}{lcccc}
\hline Style of popular music & Type 1 & Type 2 & Type 3 & Type 4 \\
Accuracy (\%) & Accuracy (\%) & Accuracy (\%) & 81 & Accuracy (\%) \\
\hline Sentimental & 81 & 89 & 93 & 93 \\
Passionate & 100 & 89 & 85 & 83 \\
Quiet & 85 & 73 & 89 & 81 \\
Lonely & 77 & 93 & 89 \\
\hline
\end{tabular}

pieces of music with similar styles, the vector sequence is not a corresponding frame, but only a table. Since the current frame indicates the time sequence in the first popular music, in order to improve the similarity of popular music, it is necessary to count the similarity count of each segment while ignoring the order of the feature vector.

The input vector is $X=\left\{x_{1}, x_{2}, \ldots, x_{n}\right\}$, $Y=\left\{y_{1}, y_{2}, \ldots, y_{n}\right\}$, the output result is $d_{\text {sum }}$, and the algorithm is described in Algorithm 1:

This algorithm describes the calculation of the distance between the element of the vector $X$ and the element of $Y$ and counts the number of minimum distances. Calculate the abovementioned distance of the sandwich cosine, and use the K-Manas method to cluster to obtain the analysis result. The results of multi-intelligence as sandwich cosine clustering are shown in Table 1. Correctly classify the four popular music styles, and display the percentage statistics in Table 2.

From the experimental results, we know that the clustering effect of popular music is better than other styles. The other three kinds of popular music are obviously different from this. The other three popular music styles are somewhat similar. There are also sad types. Please be quiet. The result was classified incorrectly. However, overall, the clustering effect is ideal. The popular music multimedia teaching path function, as a popular music perception ability required for the development of artificial intelligence, can bring users a real entertainment experience. Based on the current problems of popular music multimedia teaching path, in-depth research is conducted on traditional machine learning methods, in combination with the popular music multimedia teaching capabilities of the multiple intelligence teaching model, optimizing the multiple intelligence teaching model's role in the music multimedia teaching process, which requires more samples to find the optimal model parameters and solve the problem of the time it takes for the algorithm to run. Finally, the multiple intelligence teaching mode and traditional methods have a better effect on the time consuming of multimedia teaching.

\section{Conclusions}

In order to change the traditional educational concept, establish a new educational concept, innovate music multiple intelligences education methods, and improve the overall quality and ability of music teachers, this paper puts forward an analysis method of multimedia teaching path of popular music. By applying the multiple intelligences teaching mode to pop music multimedia teaching, this paper analyzes the actual situation of pop music teaching, summarizes the importance of integrating the quality education path in pop music teaching, discusses the ways of quality education path in pop music teaching from many aspects, better serves the needs of students' quality improvement, and obtains the optimal parameters of HMM model. The accuracy of teaching rate is improved. Finally, the results of example analysis show that the introduction of multiple intelligences teaching mode in pop music multimedia teaching, combined with the melody characteristics of pop music multimedia, can improve the accuracy of pop music multimedia teaching. In the followup, we will take music as the carrier, rich music art content, colorful music expression forms, and related music culture as students' experience, and explore the main objectives and main processes of learning.

\section{Data Availability}

The labeled datasets used to support the findings of this study are available from the corresponding author upon request.

\section{Conflicts of Interest}

The author declares no conflicts of interest. 


\section{Acknowledgments}

This study was sponsored by XinXiang University.

\section{References}

[1] Y. Ma, "Research on the arrangement and visual design of aerobics under the new situation," International Core Journal of Engineering, vol. 5, no. 9, pp. 170-173, 2019.

[2] Q. Q. Zhao and Q. Qi, "The application of computer multimedia technology in aerobics music orchestration," Applied Mechanics and Materials, vol. 651-653, pp. 1958-1961, 2014.

[3] J. Ledger, "Australian festival of chamber music wagner arrangement - conducted by $j$ ledger," Journal of Community \& Applied Social Psychology, vol. 4, no. 1, pp. 31-45, 2013.

[4] R. L. Turner, "Encyclopedia of appalachia: music2013237Encyclopedia of appalachia: music. Knoxville, TN: university of Tennessee press last visited february 2013. Gratis," Reference Reviews, vol. 27, no. 6, pp. 40-41, 2013, http://encyclopediaofappalachia.com/category.php?rec $=53$.

[5] N. Sato, "The factor of ga, ko, and zoku in the music of the ancient world: a case study upon an acculturation," Journal of Solid State Chemistry, vol. 228, no. 33, pp. 14-19, 2015.

[6] M. Fernandez-Santiago, "Of language and music: a neo-baroque, environmental approach to the human, infrahuman and superhuman in richard powers' orfeo," Anglia, vol. 137, no. 1, pp. 126-146, 2019.

[7] Julie and $\mathrm{Ra}$, "Crossing the border into 'classic music' as a 'pop musician': the case of sting," Journal of the Musicological Society of Korea, vol. 16, no. 3, pp. 11-39, 2013.

[8] D. Yue, "Misreadings in the search for commonality between Chinese and western culture," Springer Singapore, vol. 13, no. 10, pp. 6545-6553, 2016.

[9] X. Gu, Z. Y. Liu, B. Liu, B. E. Qi, and S. Wang, “A cross cultural analysis of musical timbre perception:comparison between Chinese and western culture," Journal of Clinical Otorhinolaryngology, vol. 30, no. 20, pp. 1589-1592, 2016.

[10] K. Xue and M. Yu, "New media and Chinese society, communication, culture and change in Asia," Shaping Music Consumption in China's New Media Eera: Uuse, Eexchange, and Iidentity, Springer, Manhattan, NY, USA, pp. 239-254, 2017.

[11] S. Chithra, M. S. Sinith, and A. Gayathri, "Music information retrieval for polyphonic signals using hidden Markov model," Procedia Computer Science, vol. 46, pp. 381-387, 2015.

[12] Y. Chen, "Automatic classification and analysis of music multimedia combined with hidden markov model," Advances in Multimedia, vol. 2021, no. 8, pp. 1-7, 2021.

[13] C. C. Chen, D. J. Hong, S. C. Chen, Y. Y. Shih, and Y. L. Chen, "Study of multimedia technology in posture training for the elderly," Engineering, vol. 5, no. 10, pp. 47-52, 2016.

[14] B. Kostek, "Music information retrieval-The impact of technology, crowdsourcing, big data, and the cloud in art," Journal of the Acoustical Society of America, vol. 146, no. 4, p. 2946, 2019. 